Med. thorac. 1962;19(Suppl. 1):I-IV

\title{
Contents, Vol. 19, Supplementum 1, 1962
}

\section{Medicina Thoracalis}

Vol. 19 (1962)

Internationale Zeitschrift für Erkrankungen der Thoraxorgane

International Review of Thoracic Diseases

Revue Internationale des Maladies du Thorax

Verlag S. Karger AG, Arnold Böcklinstraße 25, Basel (Schweiz)

Alle Rechte, insbesondere das der Übersetzung in fremde Sprachen, vorbehalten.

Ohøe ausdrückliche Genehmigung des Verlages ist es auch nicht gestattet, dieses Buch oder

Teile daraus

auf photomechanischem Wege (Photokopie, Mikrokopie) zu vervielfältigen.

Copyright 1963 by S. Karger AG, Basel

Printed in Switzerland by Schellenberg-Druck, Pfäffikon ZH

Cliches: Steiner \& Co. AG, Basel

INDEX

Keller, H. und Regli, J. (Heiligenschwendi):

Vorläufige Erfahrungen mit einer speziellen Kurstatioii für erwaclisene

Asthmakranke 5

Favez, G. (Lausanne):

Traitement spécifique de tuberculeux inoperables par goutte à goutte

intracavitaire 16

Arnold, E. et Hunyadi, E. (Montana):

Déclenchement d'une tuberculose evolutive après traitement cortisonique 17

A $\pi$ igiverd, R. (Zurich):

Die Mediastinoskopie, eín zielsicheres Verfahren in der Diagnostik von

Erkrankungen der Lungen und des Mediastinums 24

Sturzenegger, H. (Bern):

Uber den Bronchusstumpfverschluß bei der Lungenresektion

Taddei, M. et de Week, L. (Marsens):

Trachéobronchopathie chondroplastique. Contribution clinique, endoscopique et histologique $\quad 40$

Lapp, R, (Fribourg):

Demonstration de cas cliniques $\quad 50$

Wasserfalien, M. (Leysin):

Considerations sur le traitement actuel de la tbc ostéoarticulaire . . . 55

Vullíémoz, P., Gheorghiade, T. et Favez, G. (Lausanne):

Recherche d'un éventuel effet hépatotoxique des medicaments antibacillaires, à brève et à longue échéance 61

Gartmann, J. Ch. (Arosa):

Diagnostische Probleme des Morbus Boeck 62 
